\title{
Coherent Anti-Stokes Raman Spectroscopy
}

National Cancer Institute

\section{Source}

National Cancer Institute. Coherent Anti-Stokes Raman Spectroscopy. NCI Thesaurus. Code C160771.

A nonlinear Raman spectroscopy technique. It uses two very strong collinear lasers to irradiate a sample, instead of the traditional single laser and is orders of mag nitude stronger than spontaneous Raman emission. 